\title{
EVALUACIÓN DE LA CAPACIDAD DE IRRIGACIÓN Y DRENAJE DE TRES SONDAS DE LAVADO VESICAL
}

\author{
Ramón Diz Rodríguez, Monfragüe Pulido Díaz, Miguel Virseda Chamorro, Ignacio Arance Gil, \\ Eva Paños Facundo y José Andrés Cabrera Cabrera.
}

Servicio de Urología. Hospital Central de la Defensa. Madrid. España.

\begin{abstract}
Resumen.- OBJETIVO: Realizar una evaluación comparativa de tres tipos de sondas de lavado vesical con el fin de determinar cuál de ellas permitía un mayor flujo de entrada el líquido de lavado y de salida del líquido de la vejiga.
\end{abstract}

MÉTODOS: Se compararon tres tipos de sonda de tres vías, de un calibre 22 F, cuya principal diferencia fue el material de que estaban fabricadas: látex, silicona o polivinilo.

RESULTADOS: La sonda de polivinilo mostró una diferencia significativa tanto en el flujo de entrada como de salida del líquido de infusión respecto a los otros
Ramón Diz Rodríguez

Servicio de urología Hospital Central de la Defensa Madrid. (España). bgmeli@terra.es

Trabajo recibido: 11 de septiembre 2007 dos tipos de sonda $1 p=0,000$, ANOVA con test post hoc de Sheffe). La sonda de látex mostró además una disminución significativa del flujo de salida al inflar el balón autorretentivo.

CONCLUSIONES: La sonda de polivinilo, debido a la rigidez del material con que está fabricada, es la que un mayor flujo de lavado vesical permite.

Palabras clave: Sonda uretral. Lavado vesical. Biomateriales.

Summary.- OBJECTIVES: To perform a comparative evaluation of three types of continuous bladder irrigation catheters with the aim of determining which of them allows greater irrigation solution inflow and bladder oufflow.

METHODS: We compared three types of three-way catheters, 22F in caliber, being the material the main difference between them: latex, silicone, or polyvinyl.

RESULTS: The polyvinyl catheter showed significant differences both in inflow and oufflow in comparison with the other two types of catheters $(p=0,000$, ANOVA test with Sheffe's post hoc). Additionally, the latex catheter showed a significant oufflow decrease with the insufflation of the self retentive balloon.

CONCLUSIONS: The polyvinyl catheter, due to material rigidity, is the one that allows better bladder irrigation.

Keywords: Ureteral catheter. Bladder irrigation. Biomaterials. 


\section{INTRODUCCIÓN}

La hematuria macroscópica es una urgencia urológica frecuente sobretodo en personas de la tercera edad (1). Su tratamiento inicial incluye el lavado vesical con suero salino, con la finalidad de mantener la vía urinaria permeable, y retirar los coágulos que pueden llegar a obstruir la salida de la orina (2).

Para cumplir esta función es esencial conseguir el máximo flujo a través del catéter urinario, tanto de entrada de suero lavador, como de salida de la vejiga.

Existen en el mercado múltiples sondas vesicales con un canal de entrada (sonda de tres vías), para facilitar el lavado vesical. El objetivo de nuestro trabajo es comparar el flujo de entrada y de salida de tres tipos diferentes de sondas de lavado vesical disponibles en el mercado.

\section{MATERIAL Y MÉTODOS}

Se realizó una evaluación comparativa de tres tipos de sonda de lavado vesical (tres vías), del calibre $22 \mathrm{~F}$ :

- Sonda de látex Foley, con extremo recto tipo couvelaire (CE 0124, Ref. 9395, BSN medical GMBH D22771, Hamburg).

- Sonda semirrígida siliconada Foley, con extremo acodado tipo Dufour y punta biselada tipo Whistle (CE 0459. AB37722, Porges, S.A. La Boursidière F92357 Le Plessis Robinson Codex-France).

- Sonda de simplastic Foley, con extremo recto tipo Whistle (CE 0473. Cat no 663230 Rüsch Manufacturing (UK) Ldt. Creese Business Park High Wycombe, Bucks, HP 123 NB U.K).

Para medir el flujo de entrada del lavado vesical, se diseñó un modelo in vitro consistente en un recipiente de $500 \mathrm{ml}$ de suero salino situado a $100 \mathrm{~cm}$ del suelo, abierto por su base (para igualar su presión superficial a la atmosférica), conectado a la sonda objeto de evaluación, dejando que el suero fluyera libremente sobre un flujómetro MMS $®$ que midió el tiempo de flujo y el volumen de suero eliminado (Figura 1).

Para medir el flujo de salida de la sonda, el modelo consistió en un recipiente con $500 \mathrm{ml}$ de suero salino, en la salida del recipiente, se conectó la sonda vesical objeto de evaluación, y se dejó fluir libremente el suero salino a su través, registrándose los datos de flujo en el flujómetro (Figura 2).

Para determinar la influencia del llenado del globo vesical en los parámetros de flujo, los registros se realizaron con el globo vacío e inflado.

Previamente se realizó un estudio piloto para determinar el tamaño de la muestra necesario para detectar una variación de flujo medio de $1 \mathrm{ml} / \mathrm{s}$, con una potencia estadística del $80 \%$ y un error alfa de 0,05 , resultando ser de 6 registros por sonda.

Los resultados de la evaluación se procesaron mediante el programa estadístico SPSS. Las prue-

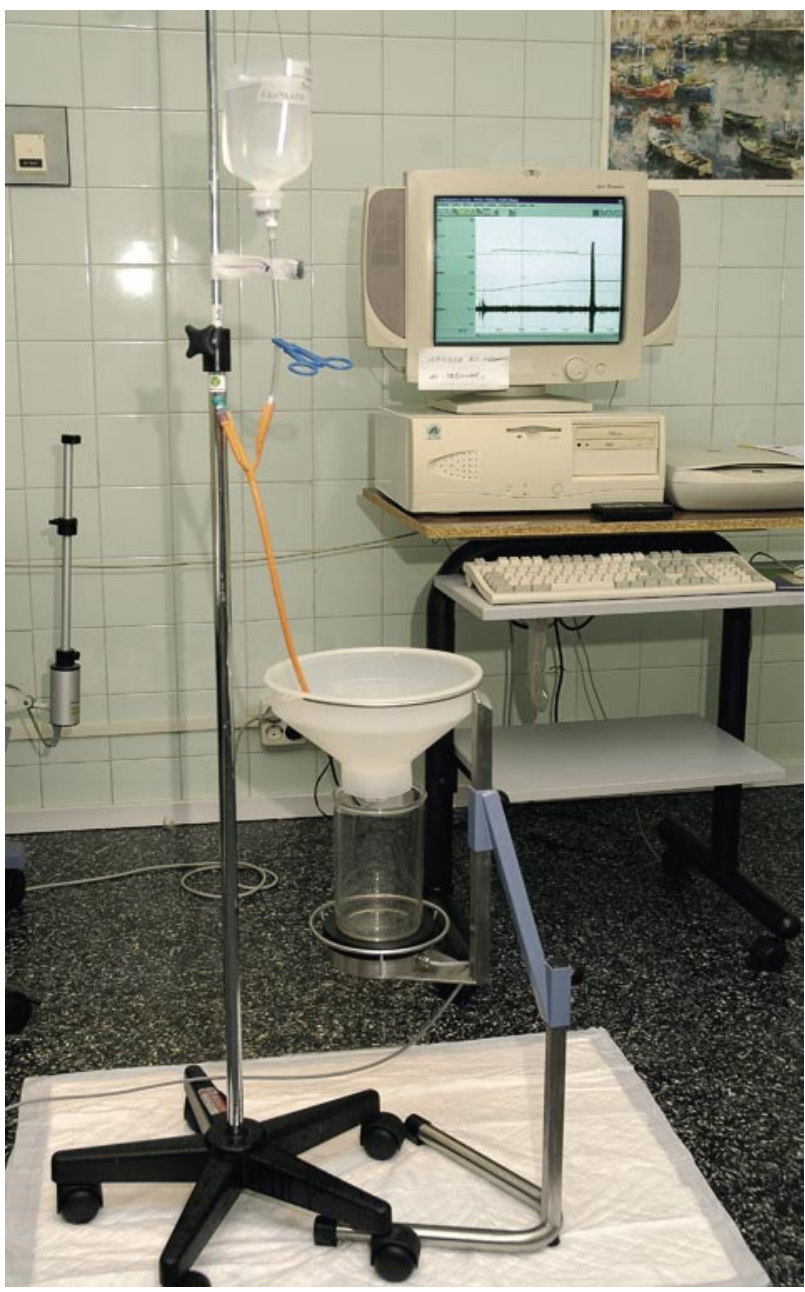

FIGURA 1. Modelo in vitro para evaluar la velocidad de entrada de la infusión salina por el canal de lavado de la sonda. Se conectó un recipiente con $500 \mathrm{ml}$ de suero salino al canal de entrada de la infusión de la sonda, y se midió el flujo de entrada, dejando caer libremente el suero salino a través de la sonda hasta un flujómetro. 


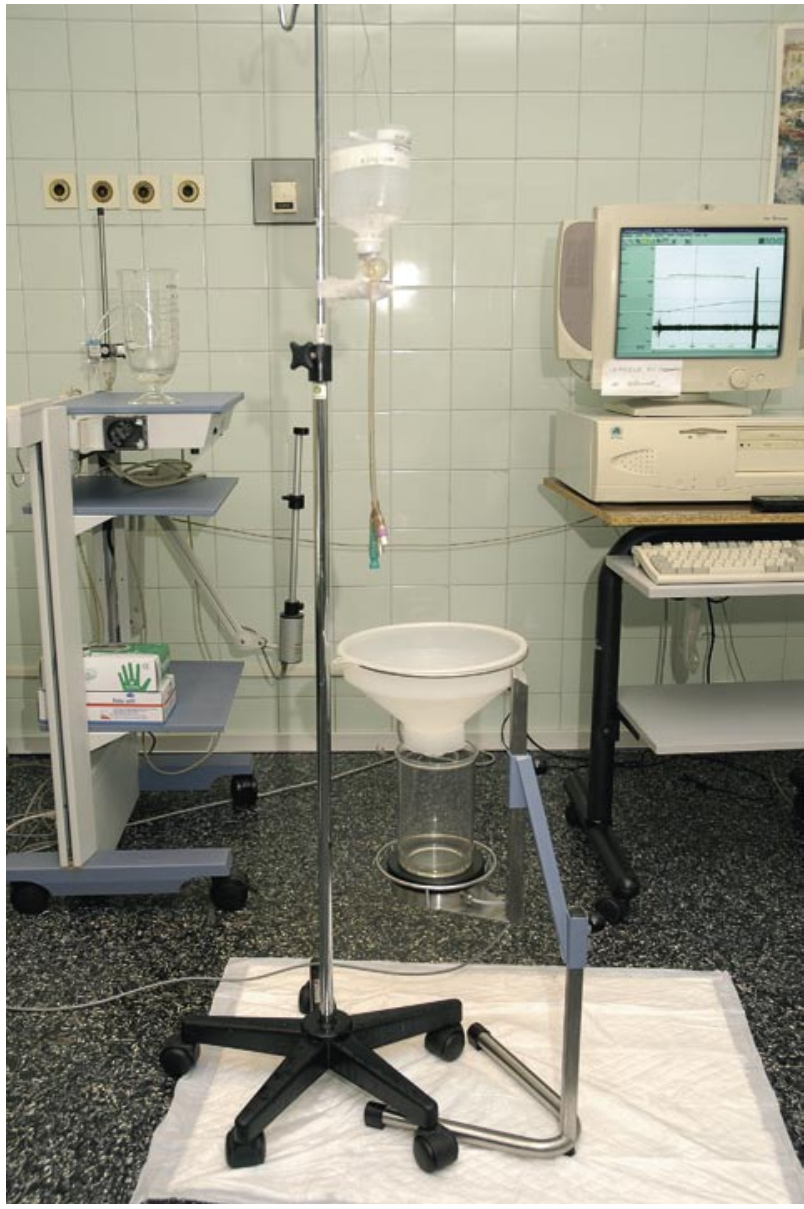

FIGURA 2. Modelo in vitro para evaluar la velocidad de salida (drenaje) de la sonda vesical. Se introdujo la sonda a través del cuello del recipiente hasta asomar en su interior, y se midió el flujo de salida a través de la sonda mediante su caída libre en el flujómetro.

bas estadísticas utilizadas fueron el test de comparación de medias de al t de Student y el análisis de la variancia (ANOVA), con el test post hoc de Scheffe. El nivel de significación se fijo en el 95\% bilateral.

\section{RESULTADOS}

\section{Análisis comparativo del flujo de entrada}

Los valores de flujo máximo y medio, con el balón uretral inflado y desinflado, de los tres tipos de sonda se muestran en la Tabla I. La única diferencia significativa observada fue en el flujo de llenado de la sonda de simplastic respecto a las otras dos sondas. Esta diferencia fue de entre 0,5 y $0,8 \mathrm{ml} / \mathrm{s}$ más de flujo de la sonda de simplastic respecto a las otras dos sondas (diferencia de medias con un $95 \%$ de confianza).

\section{Análisis comparativo del flujo de salida}

Los valores de flujo máximo y medio, con el balón uretral inflado y desinflado, de los tres tipos de sonda se muestran en la Tabla II. De nuevo se observó una diferencia significativa a favor de la sonda de simplastic. En este caso la diferencia fue mucho más amplia: entre 13 y $14 \mathrm{ml} / \mathrm{s}$ más de flujo de salida con la sonda de simplastic, respecto a las otras dos sondas (diferencias de medias con un $95 \%$ de confianza).

Además se apreció una disminución significativa del flujo de salida en la sonda de látex al inflar el balón vesical. Esta diferencia fue entre 0,2 y 0,6 $\mathrm{ml} / \mathrm{s}$ menor con el balón hinchado (diferencia de medias con un $95 \%$ de confianza).

\section{DISCUSIÓN}

La decisión sobre la sonda que mejor se adapte a las condiciones de hematuria es una de las cuestiones recurrentes en Urología (3). La evaluación de modelos in vitro, tiene la ventaja sobre los modelos reales $o$ in vivo, en que se controlan mejor las variables que pueden influir sobre el resultado de la evaluación. Braasch y cols. (4) elaboran un modelo in vitro parecido al diseñado en nuestro estudio con el que obtienen velocidades de entrada de flujo de

TABLA I. RELACIÓN DEL FLUJO MEDIO DE ENTRADA CON EL TIPO DE SONDA Y EL INFLADO DEL BALÓN.

\begin{tabular}{|l|l|l|l|}
\hline & Sonda látex* & Sonda semirrígida* & Sonda simplastic* $^{*}$ \\
\hline Sin balón & $2,00(0,26)$ & $2,06(0,01)$ & $2,68(0,004) \dagger$ \\
\hline Con balón & $2,09(0,02) \ddagger$ & $2,05(0,02) \ddagger$ & $2,75(0,05) \dagger \ddagger$ \\
\hline
\end{tabular}

* $\mathrm{ml} / \mathrm{s}$ : media (entre paréntesis desviación típica)

† Diferencias significativas ( $p=0,000)$ de la sonda de simplastic respecto de las otras dos sondas (ANOVA test post hoc de Sheffel

. f Ausencia de diferencias significativas entre cada sonda con o sin inflado del balón (t Student). 
entre 1,6 y $1,7 \mathrm{ml} / \mathrm{s}$, utilizando el flujo por gravedad o en caída libre, que llegan hasta los $29 \mathrm{ml} / \mathrm{s}$ en el caso de irrigación manual con jeringa. En nuestro estudio las velocidades de entrada de flujo en caída libre fueron algo mayores entre 2,0 y $2,75 \mathrm{ml} / \mathrm{s}$.

Respecto a los resultados de nuestra evaluación se observó que la sonda de simplastic fue superior a las otras dos sondas, tanto en la velocidad de entrada del líquido de infusión, como sobretodo en la velocidad de salida o de drenaje del recipiente. La diferencia de la sonda de simplastic respecto a las otras dos sondas fue de 0,5 y a $0,8 \mathrm{ml} / \mathrm{s}$ mayor en el caso del flujo de entrada (lo que representa entre un $25 \%$ y un $40 \%$ mas de flujo), y de entre 13 y $14 \mathrm{ml} / \mathrm{s}$ más de flujo de salida (cinco veces más flujo).

El flujo de un fluido a través de una sonda urinaria según los principios de la mecánica de fluidos derivados de la ecuación de Poiseville, depende directamente de la cuarta potencia del radio de entrada a la sonda y de la diferencia de presión entre sus extremos, e inversamente de la viscosidad del fluido y de la longitud de la sonda (5).

Las diferencias entre los tres tipos de sonda evaluados son dos: la superficie total de los orificios de salida de su extremo distal, y el tipo de material del que están fabricadas. Las tres sondas tienen su extremo distal biselado. Tanto la sonda simplastic, como la de látex son rectas, mientras que la semirrígida tiene un curvatura distal de 45 (Dufour). Además la sonda de silastic tiene otros dos orificios distales, la semirrígida tres y la de látex cuatro. La influencia del número de orificios en la velocidad del lavado vesical ha sido puesta en evidencia por algunos autores que han llegado a desarrollar una sonda de lavado con 32 orificios distales (6). Sin embargo no parece ser esta una variable que explique las diferen-

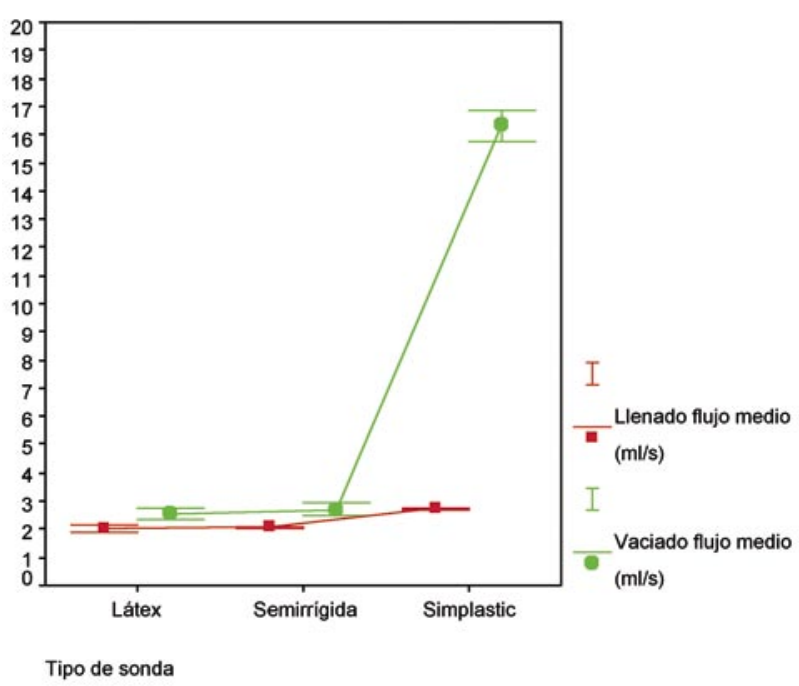

FIGURA 3. Cuadrado: valor del flujo medio de entrada con su intervalo de confianza. Círculo: valor del flujo medio de salida con su intervalo de confianza. En ordenadas flujo medio, en abcisas tipo de sonda evaluada.

cias observadas entre los tres tipos de sondas, pues la sonda de más flujo (simplastic), es la que menos orificios distales tiene.

La otra diferencia entre las tres sondas es el material de la que están fabricadas. Las sondas con base de látex tienen una mayor distensibilidad que las fabricadas con silicona que son más rígidas (7). La proporción de silicona es mayor en la sonda semirrígida y minima en la de látex, la sonda de symplastic por su parte esta fabricada con un polímero de poliuretano que le confiere mayor rigidez.

TABLA II. RELACIÓN DEL FLUJO MEDIO DE SALIDA CON EL TIPO DE SONDA Y EL INFLADO DEL BALÓN.

\begin{tabular}{|l|l|l|l|}
\hline & Sonda látex $^{*}$ & Sonda semirrígida* $^{*}$ & Sonda simplastic $^{*}$ \\
\hline Sin balón & $2,72(0,19)$ & $2,05(0,44)$ & $16,21(0,77) \dagger$ \\
\hline Con balón & $2,31(0,06) \ddagger$ & $2,55(0,27) \S$ & $16,09(0,91) \dagger \S$ \\
\hline
\end{tabular}

* $\mathrm{ml} / \mathrm{s}$ : media (entre paréntesis desviación típica)

† Diferencias significativas ( $p=0,000$ ) de la sonda de simplastic respecto de las otras dos sondas (ANOVA test post hoc de Sheffel

‡ Diferencias significativas con o sin inflado del balón ( $p=0,02$, † Student)

$\S$ Ausencia de diferencias significativas entre cada sonda con o sin inflado del balón (t Student). 
La explicación del mayor flujo conseguido con la sonda de silicona se debe a la rigidez del material. La rigidez a su vez se relaciona con el proceso de salida de aire por los orificios de la sonda y su sustitución por un volumen equivalente de líquido. Este proceso genera una presión negativa o succión que colapsa con más facilidad las sondas hechas con materiales mas distensibles como el látex, la silicona, debido a su rigidez más resistente a este fenómeno (8). La máxima rigidez se obtiene con el polímero de vinilo (9). Este fenómeno demostró en nuestro estudio ser mucho más determinante en el caso del drenaje o flujo de salida de la sonda, que en el de entrada del líquido de infusión.

Un último aspecto apuntado por algunos autores es la influencia del inflado del balón de la sonda sobre el drenaje del líquido a través de la sonda (2). En nuestro estudio, esta influencia solo se comprobó en el caso de la sonda de látex.

En conclusión, los datos del estudio in vitro, indican que la mejor sonda de lavado vesical a igualdad de diámetro y longitud, es la fabricada con polivinilo.

\section{BIBLIOGRAFÍA Y LECTURAS RECOMENDADAS (*lectura de interés $y^{* *}$ lectura fundamental)}

1. NICOLLE, L.E.; ORR, P.; DUCKWORTH, H. y cols.: "Gross hematuria in residents of long term care facilities". Am. J. Med., 94: 611, 1993.
2. CARTER, H.B.: "Instrumentation and endoscopy". Walsh, Retik, Stamey, Vaughan. Campbell's urology. W.B. Saunders Co 6th Ed., Philadelphia, 331-341, 1992.

3. GARCIA, M.M.; GULATI, S.; LIEPMANN, D. y cols.: "Traditional Foley drainage systems do they drain the bladder?". J. Urol., 177: 203, 2007.

*4. BRAASCH, M.; ANTOLAK, C.; HENDLIN, K. y cols.: "Irrigation and drainage properties of threeway urethral catheters". Urology, 67: 40, 2006.

5. LEE, K.H.; HAN, J.K.; KIM, K.G. y cols.: "Clogging of drainage catheters: Quantitative and longitudinal assessment by monitoring intracatheter pressure in catheters and rabbits". Radiology, 833-8, 2003.

6. KORTH, K.; ERSCHIG, M.; KUENKEL, M.: "The "TUR-Catheter", a new catheter for postoperative drainage of the bladder". Rev. Med. Suisse Romande, 112: 753, 1992.

*7. GLAHN, B.E.: "Influence of drainage conditions on mucosal bladder damage by indwelling catheters. I. Pressure study". Scand. J. Urol. Nephrol., 22: 93, 1988.

8. LAWRENCE, E.L.; TURNER, I.G.: "Kink, flow and retention properties of urinary catheters part 1: Conventional foley catheters". J. Mater. Sci. Mater. Med., 17:147, 2006.

*9. LAWRENCE, E.L.; TURNER, I.G.: "Kink, flow and retention properties of urinary catheters part 2: Novel design”. J. Mater. Sci. Mater. Med., 17: 153. 2006. 